\title{
HDAC3-Mediated Repression of the Nr4a Family Contributes to Age-Related Impairments in Long-Term Memory
}

\author{
- Janine L. Kwapis, ${ }^{1}$ Yasaman Alaghband, ${ }^{1}$ Alberto J. López, ${ }^{1}$ Jeffrey M. Long, ${ }^{2}$ Xiang Li, ${ }^{1}$ Guanhua Shu, ${ }^{1}$ \\ Kasuni K. Bodinayake, ${ }^{1}$ Dina P. Matheos, ${ }^{1}$ Peter R. Rapp, ${ }^{2}$ and Marcelo A. Wood ${ }^{1}$ \\ ${ }^{1}$ Department of Neurobiology and Behavior, Center for the Neurobiology of Learning and Memory, University of California, Irvine, Irvine, California 92697, \\ and ${ }^{2}$ Laboratory of Behavioral Neuroscience, National Institute on Aging, Biomedical Research Center, National Institutes of Health, Baltimore,
}

Maryland 21224

Aging is accompanied by cognitive deficits, including impairments in long-term memory formation. Understanding the molecular mechanisms that support preserved cognitive function in aged animals is a critical step toward identifying novel therapeutic targets that could improve memory in aging individuals. One potential mechanism is the Nr4a family of genes, a group of CREB-dependent nuclear orphan receptors that have previously been shown to be important for hippocampal memory formation. Here, using a cross-species approach, we tested the role of $\mathrm{Nr} 4 \mathrm{al}$ and $\mathrm{Nr} 4 \mathrm{a} 2$ in age-related memory impairments. Using a rat model designed to identify individual differences in age-related memory impairments, we first identified $\mathrm{Nr} 4 \mathrm{a} 2$ as a key gene that fails to be induced by learning in cognitively impaired male aged rats. Next, using a mouse model that allows for genetic manipulations, we determined that histone deacetylase 3 (HDAC3) negatively regulates $\mathrm{Nr} 4 \mathrm{a} 2$ in the aged male and female hippocampus. Finally, we show that overexpression of $\mathrm{Nr} 4 \mathrm{a} 1$, $\mathrm{Nr} 4 \mathrm{a} 2$, or both transcripts in the male mouse dorsal hippocampus can ameliorate age-related impairments in object location memory. Together, our results suggest that $\mathrm{Nr} 4 \mathrm{a} 2$ may be a key mechanism that promotes preserved cognitive function in old age, with HDAC3-mediated repression of $\mathrm{Nr} 4 \mathrm{a} 2$ contributing to age-related cognitive decline. More broadly, these results indicate that therapeutic strategies to promote $\mathrm{Nr} 4 \mathrm{a}$ gene expression or function may be an effective strategy to improve cognitive function in old age.

Key words: aging; epigenetics; HDAC3; memory; Nr4a1; Nr4a2

Significance Statement

Aging is accompanied by memory impairments, although there is a great deal of variability in the severity of these impairments. Identifying molecular mechanisms that promote preserved memory or participate in cognitive reserve in old age is important to develop strategies that promote healthy cognitive aging. Here, we show that learning-induced expression of the CREB-regulated nuclear receptor gene $N r 4 a 2$ is selectively impaired in aged rats with memory impairments. Further, we show that $\mathrm{Nr} 4 a 2$ is regulated by histone deacetylase HDAC3 in the aged mouse hippocampus. Finally, we demonstrate that hippocampal overexpression of either $\mathrm{Nr} 4 \mathrm{a} 2$ or its family member, $\mathrm{Nr} 4 \mathrm{al}$, can ameliorate age-related memory impairments. This suggests that promoting $\mathrm{Nr} 4 \mathrm{a}$ expression may be a novel strategy to improve memory in aging individuals.

\section{Introduction}

Normal aging is accompanied by cognitive decline, including difficulty forming and storing memories. Rather than producing

Received 0ct. 29, 2018; revised March 27, 2019; accepted April 14, 2019

Author contributions: J.L.K., D.P.M., P.R.R., and M.A.W. designed research; J.L.K., Y.A., A.J.L., J.M.L., X.L., G.S., K.K.B., and D.P.M. performed research; J.L.K. analyzed data; X.L. contributed unpublished reagents/analytic tools; J.L.K. and M.A.W. wrote the paper.

This work was supported by National Institutes of Health Grants MH101491, AG051807, and AG050787 to M.A.W., the National Institute on Aging Grants F32 AG052303 and K99 AG056596 to J.L.K., and in part by the Intramural Research Program of the National Institute on Aging. We thank Dr. Timothy Bredy for his help developing the HA-Nr4a1 virus.

The authors declare no competing financial interests.

Correspondence should be addressed to Marcelo A. Wood at mwood@uci.edu. substantial neuronal death (Rapp and Gallagher, 1996), aging typically triggers changes in intracellular signaling and impairments in learning-induced transcription (Penner et al., 2010; Spiegel et al., 2014). As de novo transcription is required for longterm memory formation (Alberini, 2009), this altered gene expression might contribute to memory impairments that occur in old age. Understanding the molecular mechanisms that contribute to dysregulated transcription in the aged brain is therefore an important step toward developing therapeutic interventions to prolong healthy cognitive aging.

https://doi.org/10.1523/JNEUROSCI.2799-18.2019

Copyright $\odot 2019$ the authors 
Transcription is controlled in part through changes in chromatin structure, which can dynamically promote or restrict access to neuronal DNA following a learning event. Numerous chromatin regulatory mechanisms have been implicated in memory (Levenson et al., 2004; Jarome et al., 2014; Kwapis and Wood, 2014), including DNA methylation, nucleosome remodeling, and multiple histone modifications (e.g., acetylation, methylation, phosphorylation). Histone acetylation has received the most attention as a mechanism involved in age-related cognitive decline, with work from our laboratory (Kwapis et al., 2018) and others (Peleg et al., 2010; Reolon et al., 2011; Castellano et al., 2012; Benito et al., 2015; Sharma et al., 2015) demonstrating that altered histone acetylation is associated with reduced memory performance in old age. We recently demonstrated that histone deacetylase 3 (HDAC3), which represses histone acetylation and memory formation (McQuown et al., 2011; Malvaez et al., 2013; Bieszczad et al., 2015; Alaghband et al., 2017), contributes to age-related impairments in hippocampal memory (Kwapis et al., 2018). HDAC3 deletion improves hippocampal memory and restores expression of a subset of learning-induced genes, including one member of the Nr4a family, Nr4a1 (Kwapis et al., 2018).

The Nr4a family consists of three genes: Nr4al (NGFI-B, NUR77, TR3), Nr4a2 (NURR1, HZF-3, RNR1), and Nr4a3 (NOR1, MINOR, TEC). Each is a transcription factor and immediate early gene (IEG) that is activated by signaling cascades important for long-term memory formation, including cAMP responsive element binding protein (CREB; for review, see Hawk and Abel, 2011). The Nr4a family has been implicated in hippocampal synaptic plasticity (Bridi et al., 2017) and long-term memory formation (Peña de Ortiz et al., 2000; Hawk et al., 2012; McNulty et al., 2012; Malvaez et al., 2013; Rogge et al., 2013). Both NR4A1 and NR4A2 are required for hippocampusdependent long-term memory (Peña de Ortiz et al., 2000; Hawk et al., 2012; McNulty et al., 2012) and are intimately connected with HDAC activity; not only is transcription of both genes regulated by HDACs (Vecsey et al., 2007; Hawk et al., 2012; Bridi et al., 2017), their expression is required for memory enhancements induced by HDAC inhibition (McQuown et al., 2011; Hawk et al., 2012; Bridi et al., 2017). Nr4a1 and Nr4a2 are therefore modulated by HDAC expression and critical for long-term memory formation.

In this study, we tested the hypothesis that HDAC3-mediated inhibition of Nr4a1 and Nr4a2 contributes to age-related hippocampal memory impairments. As Nr4a2 was previously identified as a target of HDAC3 (McQuown et al., 2011; Malvaez et al., 2013; Rogge et al., 2013) and can function synergistically with Nr4a1 to promote transcription (Maira et al., 1999; Hawk and Abel, 2011), we anticipated that simultaneous overexpression of both transcripts would ameliorate age-related impairments in long-term memory. Here, we used a cross-species approach to take advantage of a well characterized rat model designed to detect individual differences in age-related cognitive decline (for review, see Gallagher et al., 2006) and a mouse model that allows for conditional, site-specific genetic knock-out of Hdac3. We found that learning-induced $\mathrm{Nr} 4 a$ expression fails in memoryimpaired aged mice and rats. Deletion of HDAC3 restores expression of Nr4a2, but not Nr4a1 in the aged hippocampus, but overexpression of either $\mathrm{Nr} 4 \mathrm{a} 1$ or $\mathrm{Nr} 4 \mathrm{a} 2$ improves long-term object location memory. Together, these results suggest that HDAC3-mediated regulation of the Nr4a family of genes contributes to age-related impairments in hippocampal memory across species.

\section{Materials and Methods}

Subjects. Both rats and mice were used in the current study. Rats were all male Long-Evans, either young adults [between 6 and 8 months at the time of object location memory (OLM) training] or aged (between 24 and 26 months old). Rats were individually housed and maintained under a $12 \mathrm{~h}$ light/dark cycle at the National Institute on Aging (NIA) animal facilities until the completion of AU/AI (Aged Unimpaired/Aged Impaired) categorization. Following categorization as AU/AI, rats were shipped to the University of California, Irvine, where they continued to be maintained on a $12 \mathrm{~h}$ light/dark cycle.

Mice were either male C57BL/6J (see Figs. $3 A-C$, 4) or male and female HDAC $3^{\text {flox/flox }} / \mathrm{HDAC} 3^{+/+}$littermates maintained on a C57BL/6J background (Fig. 3D-H). All mice were individually housed $1-2$ weeks before behavior and were maintained on a $12 \mathrm{~h}$ light/dark cycle. Young adult mice were between 2 and 4 months old at the time of training and aged mice were between 18 and 20 months old.

All animals had ad libitum access to food and water and all behavioral testing was performed during the light portion of the cycle. All experiments were conducted according to the U.S. National Institutes of Health guidelines for animal care and use and were approved by the Institutional Animal Care and Use Committee of the University of California, Irvine, or the NIA Animal Care and Use Committee.

Behavioral characterization of rats as aged unimpaired or aged impaired. All rats were initially trained and tested in a well characterized water maze task to categorize aged animals as either AU or AI as previously described (Gallagher et al., 1993). Rats were given 8 consecutive days of training, with three training trials per day interspersed with four probe trials (last trial of every other day). A learning index (LI) score was calculated for each rat based on the weighted average proximity (in $\mathrm{cm}$ ) to the hidden escape location across the last three probe trials. This LI measure has provided a reliable metric for classifying aged rats as either $\mathrm{AU}$ or $\mathrm{AI}$ in previous research (Gallagher et al., 1993; Koh et al., 2010; Spiegel et al., 2013; Tomás Pereira et al., 2013; Ash et al., 2016). Rats were tested in a single cued session of the hippocampus-independent cued water maze task the following day and animals that perform outside of the normal range on this task are excluded from analyses. Following categorization, rats were shipped to the University of California, Irvine, for further behavioral and molecular analyses. Rats were all given 2-4 weeks to acclimate after arrival at UCI before we began handling for OLM.

Surgery. All mice received surgery 2 weeks before behavior, as described previously (White et al., 2016; Alaghband et al., 2018; Kwapis et al., 2018; Lopez et al., 2018). Briefly, mice were anesthetized with isoflurane, placed in a stereotaxic apparatus, and injection needles were slowly lowered to the dorsal hippocampus $(0.2 \mathrm{~mm} / 15 \mathrm{~s})$. The final coordinates used were as follows: AP: $-2.0 \mathrm{~mm}, \mathrm{ML}: \pm 1.5 \mathrm{~mm}, \mathrm{DV}:-1.5 \mathrm{~mm}$ relative to bregma. Virus was bilaterally injected slowly $(10 \mathrm{~min} / \mu \mathrm{l})$, needles remained in place for an additional $2 \mathrm{~min}$, and injection needles were then slowly removed. For overexpression of $\mathrm{Nr} 4 \mathrm{a} 1, \mathrm{Nr} 4 \mathrm{a} 2$, or both, mice were randomly assigned to injection conditions. For HDAC $3^{+/+}$ and HDAC $3^{\text {flox/flox }}$ mice, all animals were infused with AAV-CaMKIICre. For all behavioral experiments, animals within each viral condition were randomly assigned to home-cage/trained groups and all conditions (objects, boxes, etc.) were counterbalanced between groups.

AAV production. AAV2.1-CaMKII-Cre was purchased from the Penn Vector Core (titer: $1.81 \times 10^{13} \mathrm{GC} / \mathrm{ml}$ ). For AAV-HA-Nr4al and AAV-v5$\mathrm{Nr} 4 \mathrm{a} 2$, we amplified wild-type $\mathrm{Nr} 4 \mathrm{a} 1$ and $\mathrm{Nr} 4 \mathrm{a} 2$ from hippocampal cDNA and cloned the product into a modified backbone under the control of the $0.4 \mathrm{kB}$ CaMKII promoter and B-globin intron. A v5 tag was added to Nr4a2 and a HA tag was added to Nr4al to allow for C-terminal fusion to each construct, respectively. For the empty vector control, the coding sequence of Nr4a1 or Nr4a2 was not present, but the plasmid was otherwise identical. AAV was made by the Penn Vector Core (AAV1-v5-Nr4a2) or in Dr. Tim Bredy's laboratory at the University of California, Irvine (AAV1/2-HANr4a1) as previously described (Leighton et al., 2018). For animals infused with both AAV-HA-Nr4a1 and AAV-v5-Nr4a2, viruses were mixed in equal parts before infusing $1 \mu \mathrm{l}$ of the viral mixture into the dorsal hippocampus.

OLM and object recognition memory. The OLM and object recognition memory (ORM) tasks were conducted as previously described (Vogel- 
Ciernia and Wood, 2014; Kwapis et al., 2018). Mice were handled for 2 min per day for $4 \mathrm{~d}$ and then were given $6 \mathrm{~d}$ of habituation, whereas rats were given $3 \mathrm{~d}$ of handling followed by $3 \mathrm{~d}$ of habituation. During habituation, mice or rats were placed in the training context in the absence of objects. During training, the animals were exposed to two identical objects and allowed to explore for $10 \mathrm{~min}$. The following day, animals were given a retention test in which one object was moved to a new location (OLM) or one object was swapped with a novel item (ORM). Habituation for ORM began at least 1 week after the completion of OLM and a new context and unfamiliar objects were used (Vogel-Ciernia and Wood, 2014; Kwapis et al., 2018). Preference for the novel item was expressed as a discrimination index (DI): DI $=\left(t_{\text {novel }}-t_{\text {familiar }}\right) /\left(t_{\text {novel }}+t_{\text {familiar }}\right) \times$ $100 \%)$. Rodents that explored both objects for less than $2 \mathrm{~s}$ during testing or $3 \mathrm{~s}$ during training were removed from further analysis. Training and testing exploration were scored by hand from video recordings and all scoring was performed by experimenters blinded to experimental groups. For molecular studies, home-cage control animals were killed between trained groups in a counterbalanced fashion.

Quantitative RT-PCR. RT-qPCR was performed as described previously (Vogel-Ciernia et al., 2013; Kwapis et al., 2018). One millimeter punches were collected from area CA1 of the dorsal hippocampus in a $500 \mu \mathrm{m}$ slice of tissue. RNA was isolated from punches using an RNeasy Minikit (Qiagen) and cDNA was created using the Transcriptor First Strand cDNA Synthesis kit (Roche Applied Science). The following primers were used, designed using the Roche Universal Probe Library: Rat Nr4a1: forward primer $\left(5^{\prime}-3^{\prime}\right)$ : AGCTTGGGTGTTGATGTTCC, reverse primer $\left(5^{\prime}-3^{\prime}\right)$ : ACAGCTAGCAATGCGGTTC, probe, AGG AGCTG. Rat Nr4a2: forward primer $\left(5^{\prime}-3^{\prime}\right)$ : CCACGTCGACTCCAA TCC, reverse primer $\left(5^{\prime}-3^{\prime}\right)$ : TAGTCAGGGTTTGCCTGGAA, probe CAGCCTGG. Rat $c F o s$ : forward primer $\left(5^{\prime}-3^{\prime}\right)$ : CCCCTGTCAACACA CAGGA, reverse primer $\left(5^{\prime}-3^{\prime}\right)$ : GACCAGAGTGGGCTGCAC, probe: CTCCACCA. Mouse Nr4a1: forward primer $\left(5^{\prime}-3^{\prime}\right)$, AGCTTGGGTGT TGATGTTCC; reverse primer $\left(5^{\prime}-3^{\prime}\right)$, AATGCGATTCTGCAGCTCTT, probe, TCTGGTCC. Mouse Nr4a2: forward primer $\left(5^{\prime}-3^{\prime}\right)$, TTGCA GAATATGAACATCGACA; reverse primer $\left(5^{\prime}-3^{\prime}\right)$, GTTCCTTGAGC CCGTGTCT, probe, TTCTCCTG. mouse HA-Nr4a1: forward primer $\left(5^{\prime}-3^{\prime}\right)$, CCATACGACGTCCCAGACTAC, reverse primer $\left(5^{\prime}-3^{\prime}\right)$ : CTCGTTGCTGGTGTTCCATA, probe, CTCCTCCA. For the $v 5-\mathrm{Nr} 4 a 2$ transcript, as no Universal Probe Library assay was available, we designed a PrimeTime qPCR assay (IDT): forward primer $\left(5^{\prime}-3^{\prime}\right)$ : CATGGGTA AGCCTATCCCTAAC, reverse primer $\left(5^{\prime}-3^{\prime}\right)$ : TCTCCCGAAGAGTG GTAACT, probe: 6-FAM/TC-TCCTCGGTC/Zen/TCGATTCTACGCC TT-3lABkFQ. All of the probes for these target genes were conjugated to the dye FAM. Gapd or Hprt5 was used as a reference gene for RT-qPCR assays. For rat Gapd, we used the following primers: forward primer $\left(5^{\prime}-3^{\prime}\right)$; CTGCACCACCAACTGCTTAG, reverse primer $\left(5^{\prime}-3^{\prime}\right)$ : TGATGGCATGGACTGTGG, probe, TTGGCATCGTG. For mouse Gapd, we used the following primers: left primer, 5'-ATGGTGAAGGTCGGTGTGA-3'; right primer, 5'-AATCTCCACTTTGCCACTGC-3'; probe, TGGCGGTATTGG. For mouse Hprt5, we used the following primers: forward primer $\left(5^{\prime}-3^{\prime}\right)$; TGCTCGAGATGTCTGAAGG, reverse primer $\left(5^{\prime}-3^{\prime}\right)$ : CTTTTATGTCCCCCGTTGAC, probe, ATCACATTGTGGCCCCTCTGT. Gapd and Hprt5 probes were conjugated to LightCycler Yellow 555 to allow for multiplexing in the Roche LightCycle 480 II machine (Roche Applied Sciences). All values were normalized to Gapd or Hprt5 expression levels and each trained group was compared with a home-cage control from the same cohort to normalize any gene induced nonspecifically by transportation or handling stress. Analyses and statistics were performed using the Roche proprietary algorithms and REST 2009 software based on the Pfaffl method (Pfaffl, 2001, 2002).

Chromatin immunoprecipitation. Chromatin immunoprecipitation (ChIP) was performed on punches from area CAl of the dorsal hippocampus using the protocol from the Millipore ChIP kit as previously described (Kwapis et al., 2018). Following cross-linking with 1\% formaldehyde (Sigma-Aldrich), tissue was lysed and sonicated. Chromatin was immunoprecipitated overnight at $4^{\circ} \mathrm{C}$ with $2 \mu \mathrm{l}$ of anti-H4K8Ac (Millipore) or $2 \mu \mathrm{l}$ of Normal Rabbit Serum (negative control; Millipore). Chromatin was washed and then eluted from the beads and reverse cross-linked in the presence of proteinase K. DNA was columnpurified and $\mathrm{qPCR}$ was run using primer sequences designed by the Primer 3 program. Primers used were as follows: Nr4a1: Forward primer: 5'-GATAGAGGGGTGGCTGAAG-3', Reverse primer: 5' -AAAAGAGC TCAGTCCGACGA-3'. Nr4a2: Forward primer: 5'-TGAAGTCCGTG GTGATGCTA-3', Reverse primer: 5' -CGGGACAACTGTCTCCACTT$3^{\prime}$. Five microliters of input, anti-H4K8Ac, or anti-rabbit IgG immunoprecipitate (IP) from each animal was examined in duplicate. We used the percentage input method to normalize ChIP-qPCR data, in which the input was adjusted to $100 \%$ and both the IP and IgG samples were calculated as a percentage of this input using the formula $100 \times$ $\mathrm{AE}^{\wedge}$ [adjusted input $-C t(\mathrm{IP})$ ]. Fold enrichment was then calculated by normalizing each group to the home-cage control. An in-plate standard curve determined amplification efficiency.

Experimental design and statistical analysis. Sample sizes were similar to those generally used in the field, including those reported in previous publications (Vogel-Ciernia et al., 2013; Kwapis et al., 2018; Lopez et al., 2018) although no statistical methods were used to predetermine sample sizes. All behavior was recorded for offline analysis. For water-maze training and AU versus AI categorization, videos were scored to calculate a weighted average proximity in $\mathrm{cm}$ to the hidden escape platform across the final three probe trials, as described in detail previously (Gallagher et al., 1993). Aged rats with an LI of 240 or higher were categorized as AI.

For OLM and ORM, videos were manually scored off-line to determine exploration time for the two objects present during training or testing. All experimenters were blind to the group assignments when scoring. Exploration was scored when the animal's head was oriented toward the object within $1 \mathrm{~cm}$ or with the nose touching the object and the DI was calculated as described above. Mice that showed a preference for one object during training (DI $> \pm 20$ ) was excluded from further analysis. Additionally, any mouse that explored the objects for $<2 \mathrm{~s}$ during testing or $3 \mathrm{~s}$ during training was removed.

Statistical analyses were performed using either a two-tailed Student's $t$ test (Fig. $1 A$ ), a Pearson correlation (Fig. $1 E$ ), one-way ANOVAs (Figs. $1 C, D, 4 B-I$ ) or two-way ANOVAs (Figs. 2, 3) followed by Sidakcorrected $t$ tests to compare individual groups. Two-way ANOVAs had factors of Training and Age (Figs. 2, 3B,C) or Training and Genotype (Fig. $3 E-H$ ). qPCR and ChIP results were normalized to the mean of each home-cage control group, except for Figure $4 B-E$, in which samples are normalized to the mean of the "Both" condition. We chose to normalize to the Both condition for these analyses because the empty vector control showed virtually no expression of the injected viruses, making normalization to the empty vector (EV) control group impossible. All statistics were performed with GraphPad Prism 7 software. Main effects and interactions for all ANOVAs are described in the text, along with the specific number of animals of each sex used in each individual experiment. All analyses were two-tailed and required an $\alpha$ value of 0.05 for significance. Error bars in all figures represent SEM. For all experiments, values $\pm 2 \mathrm{SD}$ from the group mean were considered outliers and were removed from analyses.

\section{Results \\ Aged rats show individual differences in spatial learning across tasks}

To understand the role of $N r 4 a 1$ and $N r 4 a 2$ in age-related hippocampal memory impairments, we first used a well characterized rat model to identify individual differences in spatial memory decline in old age using a standardized version of the Morris water maze (Gallagher et al., 2006; Fig. 1A). Aged rats showed a significantly higher average LI in this task (indicating worse memory performance) compared with young rats (twotailed Student's $t$ test: $t_{(24)}=2.07, p=0.0499$ ) and increased individual differences among scores, consistent with previous reports (for review, see Gallagher et al., 2006). Within this group of aged rats, animals were characterized as AU if they performed similarly to young rats (range $=146-238$, mean \pm SEM $=201 \pm$ 

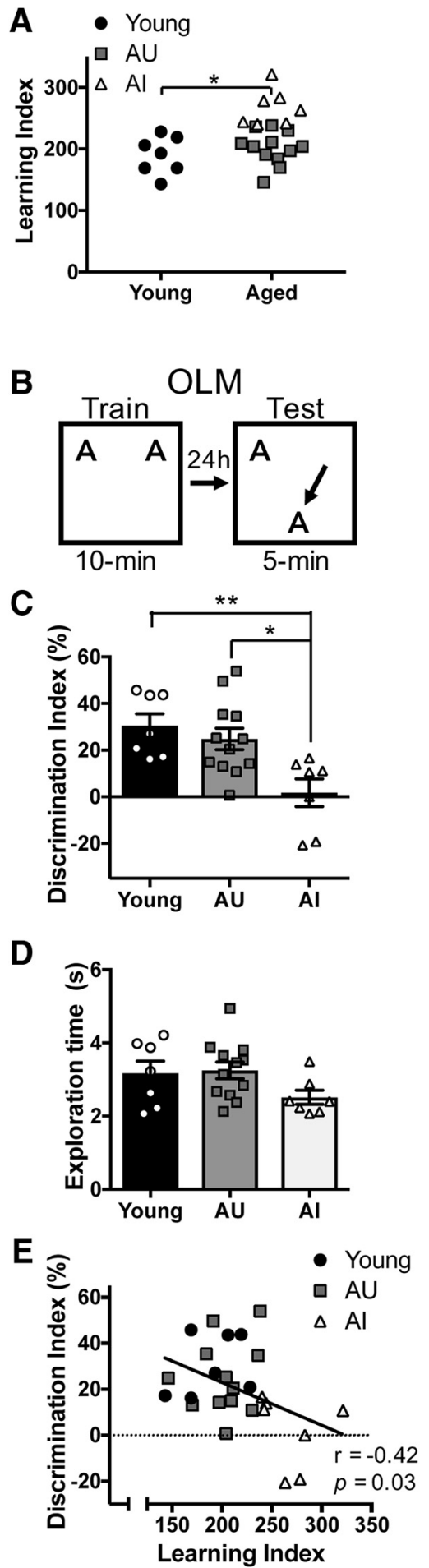

Figure 1. Behavioral characterization of young, $A U$, and Al rats. $A, L I$ scores from young, $A U$, and $A \mathrm{l}$ rats. Aged rats show significantly higher $\mathrm{LI}$ scores and a larger distribution of scores compared with young rats. $B, 0 \mathrm{LM}$ experimental design. $\boldsymbol{C}$, Young and AU rats show significantly better memory for $0 \mathrm{LM}$ than Al rats. $\boldsymbol{D}$, No group differences were observed in total object exploration time. $\boldsymbol{E}$, LI scores in the water maze negatively correlate with DI scores in OLM. All data are shown as mean \pm SEM. ${ }^{*} p<0.05,{ }^{* *} p<0.01$.

7.8). Aged rats with an LI of 240 or higher were categorized as AI $($ range $=240-321$, mean \pm SEM $=267.3 \pm 11.1)$.

To determine whether these categorizations are valid across spatial memory tasks, we next tested these rats in the OLM task. OLM is a simple incidental learning task that produces robust
A

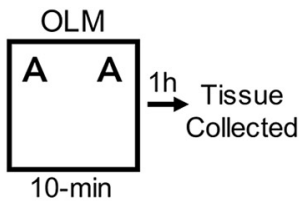

B Nr4a1

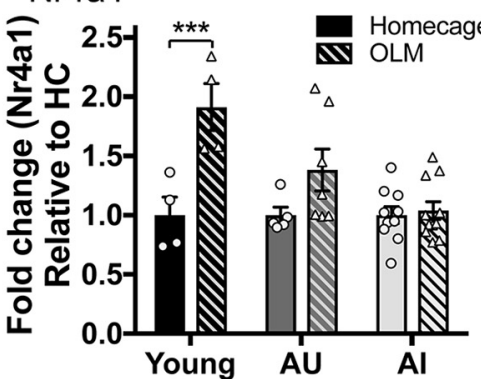

C Nr4a2
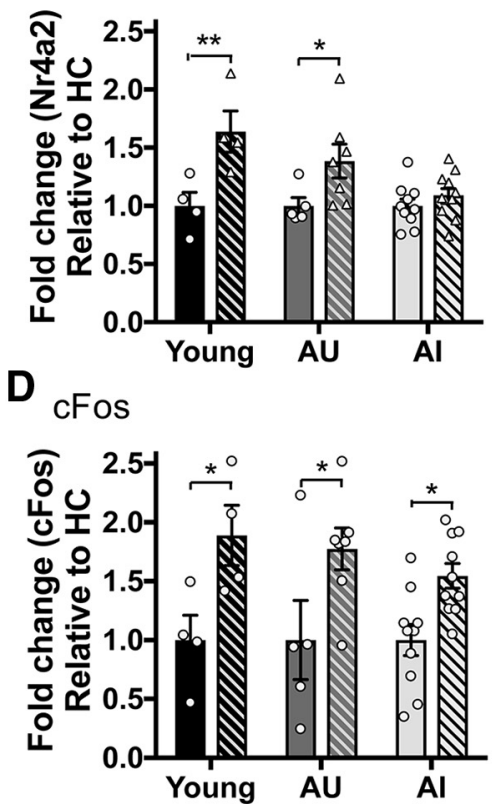

Figure 2. Learning-induced hippocampal Nr4a2 is impaired in cognitively impaired aged rats. $A$, Experimental design. Young, $\mathrm{AU}$, or $\mathrm{Al}$ rats were killed $1 \mathrm{~h}$ after $0 \mathrm{LM}$ or were killed from the HC. $\boldsymbol{B}, N r 4 a 1 \mathrm{mRNA}$ is upregulated in the dorsal hippocampus following OLM in young, but not AU or Al rats. C, Nr4a2 mRNA is upregulated by OLM in the dorsal hippocampus of young and $\mathrm{AU}$ rats but fails to increase following learning in Al rats. $\boldsymbol{D}$, Hippocampal cFos expression is significantly increased by $0 \mathrm{LM}$ in young, $\mathrm{AU}$, and $\mathrm{Al}$ rats. All data are shown as mean $\pm \mathrm{SEM}$. ${ }^{*} p<0.05,{ }^{* *} p<0.01,{ }^{* * *} p<0.001$.

long-term memory in rodents following a 10-min training session (Stefanko et al., 2009; Roozendaal et al., 2010; Barrett et al., 2011; Reolon et al., 2011; Vogel-Ciernia et al., 2013). Here, rats previously categorized as AU or AI were given 10 min OLM training in which they were exposed to two identical objects in a familiar context (Fig. 1B). The following day, memory was tested by moving one of the objects to a new location and measuring the time spent with each object. As rodents prefer novelty, memory for OLM is demonstrated by increased exploration of the object in a new location (indicated by a higher score on the DI).

OLM performance confirmed the categorization of each group as either AU or AI. Young rats showed significant preference for the moved object (one-sample $t$ test compared with 0 , $t_{(6)}=6.06, p=0.0009, n=7$, all male), indicating robust memory for training. Similarly, rats categorized as AU showed a sig- 
nificant preference for the moved object (one-sample $t$ test compared with $0, t_{(11)}=5.38, p=0.0002, n=12$, all male), comparable to the preference observed in young rats (Fig. $1 C$; one-way ANOVA, $F_{(2,23)}=7.27, p=0.0036$, Sidak's post hoc test comparing young to AU, $p=0.82)$. Rats categorized as AI, on the other hand, failed to show a significant preference for the moved object (one-sample $t$ test compared with $0, t_{(6)}=0.297, p=0.78$, $n=7$, all male) and showed a DI significantly lower than that of the young (Fig. 1C; Sidak's post hoc test, $p=0.005$ ) and AU (Sidak's post hoc test, $p=0.013$ ) rats. No differences in total exploration were observed between groups (Fig. $1 D$; one-way ANOVA, $\left.F_{(2,23)}=2.28, p=0.125\right)$.

Finally, we tested whether there was a correlation between LI and DI scores (Fig. 1E). We observed a significant inverse correlation between LI and DI scores (Pearson correlation, $r=-0.42$, $p=0.032$ ), indicating that rats with lower scores (better performance) in the water maze task also tended to show stronger preference for the moved object in OLM. Together, these results demonstrate that $\mathrm{AU}$ and $\mathrm{AI}$ categorization is consistent across spatial memory tasks.

\section{Cognitively impaired aged rats show failed induction of $\mathrm{Nr} 4 \mathrm{a} 2$ in the dorsal hippocampus following OLM training}

Previous work from our laboratory has suggested that aging is accompanied by dysregulation of $\mathrm{Nr} 4 \mathrm{a}$ gene expression (Kwapis et al., 2018). To determine whether learning-induced dysregulation of $\mathrm{Nr} 4 \mathrm{a}$ gene expression accompanies age-related cognitive impairments, we measured $N r 4 a 1$ and $N r 4 a 2$ expression during memory consolidation for OLM in young, AU, and AI rats. Following characterization in the water maze task, a new cohort of young, AU, and AI rats was trained in OLM and killed $60 \mathrm{~min}$ following the end of training (Fig. 2A), a time point at which both Nr4a1 and Nr4a2 transcripts are typically upregulated in young mice (Hawk et al., 2012). Home-cage (HC) rats were treated identically, except that they received no training session and were killed between groups in a counterbalanced fashion. Nr4a1 was significantly increased in the dorsal hippocampus of young rats following OLM training (two-way ANOVA, significant age group $\times$ training interaction; Fig. $2 B ; F_{(2,35)}=6.097, p=0.005$, Sidak's post hoc comparing young HC to young OLM, $p=0.0005$, $n=4,4$, all male)), consistent with previous reports of learninginduced Nr4al during hippocampal memory consolidation (Peña de Ortiz et al., 2000; von Hertzen and Giese, 2005; Hawk et al., 2012; McNulty et al., 2012). This learning-induced increase in Nr4a1 was blunted in both groups of aged rats; no significant increases in $\mathrm{Nr} 4 a 1$ expression were observed in either AU (Sidak's post hoc, $p=0.113, n=5,7$, all male) or AI (Sidak's post hoc, $p=0.988, n=10,11$, all male) rats. AU rats did show a trend toward upregulation of $\mathrm{Nr} 4 \mathrm{a} 1$ expression in response to learning, similar to the increase observed in young rats, but this increase was not statistically significant $(p=0.113)$. Nr4a1 expression is therefore upregulated during memory consolidation in young rats but this learning-induced increase is impaired in aged rats regardless of cognitive status.

We next measured $\mathrm{Nr} 4 a 2$ expression in these samples. Nr4a2 mRNA was significantly increased in the dorsal hippocampus of both young rats (Fig. 2C; two-way ANOVA, significant age group $\times$ training interaction: $F_{(2,35)}=3.782, p=0.033$, Sidak's post hoc comparing young HC to young OLM, $p=0.003, n=4,4$, all male) and AU rats (Sidak's post hoc, $p=0.038, n=5,7$, all male) following OLM. In contrast, Nr4a2 levels were not significantly increased in the dorsal hippocampus of AI rats (Sidak's post hoc, $p=0.792, n=10,11$, all male). Thus, learning-induced expression of $\mathrm{Nr} 4 \mathrm{a} 2$ fails in the cognitively impaired aged brain but remains intact in the aged brain with intact cognitive function.

Finally, we measured cFos expression in these samples to determine whether other IEGs in addition to those in the Nr4a family might be altered in the AI hippocampus. We found that cFos expression was induced by learning in all three groups, regardless of age or cognitive status (Fig. 2D; two-way ANOVA, significant main effect of training: $F_{(2,34)}=21.32, p<0.0001$; but no significant effect of age and no significant interaction: $n=$ $4,4,5,7,10,10$, all male). As $c$ Fos is often used as a marker of activity after learning, this indicates that OLM training drives neuronal activity in the hippocampus, even in rats with age-related memory impairments. Further, this demonstrates that some genes show normal learning-induced increases in the aged brain, even in the face of cognitive impairments. Together, these results suggest that $\mathrm{Nr} 4 a 2$ impairments, which are unique to aged rats with cognitive decline, may be a novel mechanism contributing to age-related memory decline.

\section{Learning-induced $\mathrm{Nr} 4 \mathrm{a} 2$ is also impaired in the dorsal hippocampus of aging mice}

A previous RNA sequencing study from our laboratory identified Nr4al as a key gene that is impaired in the hippocampus of old mice with memory impairments (Kwapis et al., 2018). Here, using RT-qPCR, we observed that Nr4a2, but not Nr4a1, was selectively repressed in the hippocampus of aged rats with cognitive impairments (we discuss the discrepancy in the discussion). To determine whether Nr4a1, Nr4a2, or both genes are impaired in the dorsal hippocampus of old mice, we next measured learninginduced Nr4a mRNA expression in both young (3-month-old) and old (18-month-old) wild-type mice during object location memory consolidation (Fig. $3 A$ ). Work from our laboratory has previously demonstrated that 18-month-old wild-type mice show severe deficits in OLM following 10 min training (Kwapis et al., 2018). Both trained groups were compared with HC control groups of the same age that were treated identically except that they received no training session and were killed between groups in a counterbalanced manner.

Using RT-qPCR, we found that learning-induced increases in Nr4a2, but not Nr4a1, were impaired in the dorsal hippocampus of aged mice. Nr4a1 mRNA was significantly increased by learning in the hippocampus of both young and old mice (Fig. 3B; two-way ANOVA, significant main effect of training: $F_{(1,30)}=$ $26.08, p<0.0001$; no significant effect of genotype or significant interaction, Sidak's post hoc tests comparing Young HC vs OLM: $p=0.0009$, Old HC vs OLM: $p=0.005, n=8,9,8,9$, all male). Thus, even though 18-month-old mice show age-related deficits in long-term object location memory, $\mathrm{Nr} 4 a 1$ mRNA is normally induced by OLM training. Nr4a2 mRNA, in comparison, was significantly increased by OLM in the young hippocampus but this induction failed in the old hippocampus (Fig. 3C; two-way ANOVA, significant main effect of training: $F_{(1,31)}=15.58, p=$ 0.0004 ; no significant effect of genotype or significant interaction, Sidak's post hoc tests comparing Young HC vs OLM: $p=$ 0.001 , Old HC vs OLM: $p=0.18, n=8,9,9,9$, all male). These results demonstrate that learning-induced expression of $\mathrm{Nr} 4 \mathrm{a} 2$, but not Nr4a1, is impaired in the dorsal hippocampus of aged mice. Together with Figure 2, these findings demonstrate that Nr4a2 mRNA is impaired in the dorsal hippocampus of both mice and rats showing age-related cognitive deficits. 
A
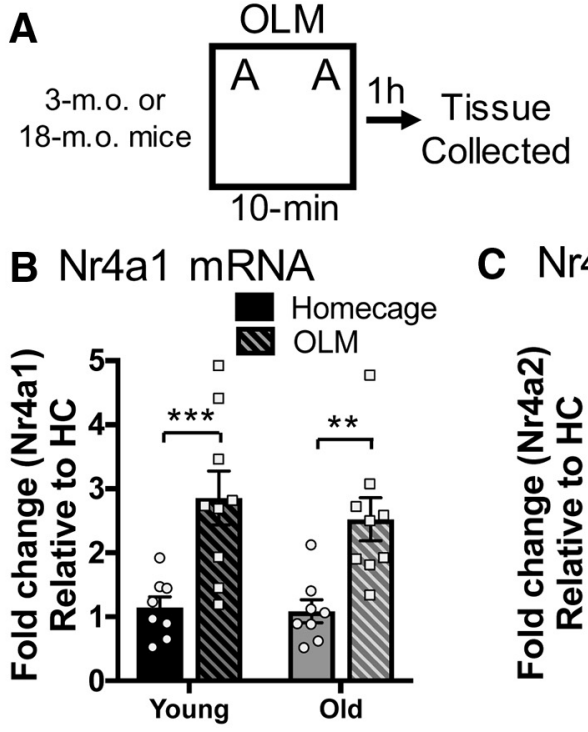

C Nr4a2 mRNA
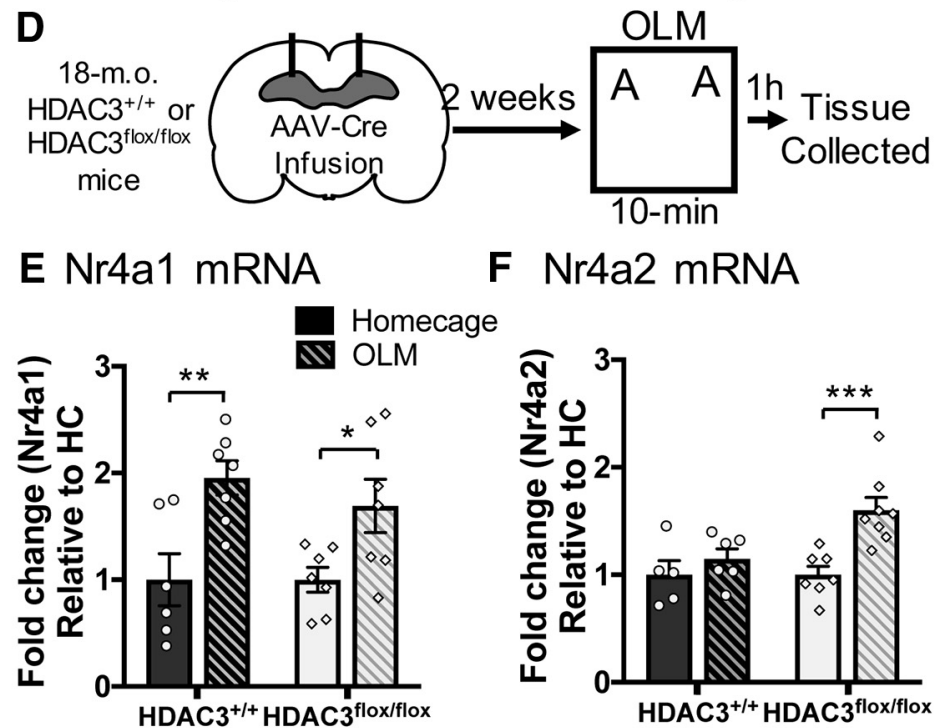

G H4K8Ac IP: Nr4a1 promoter

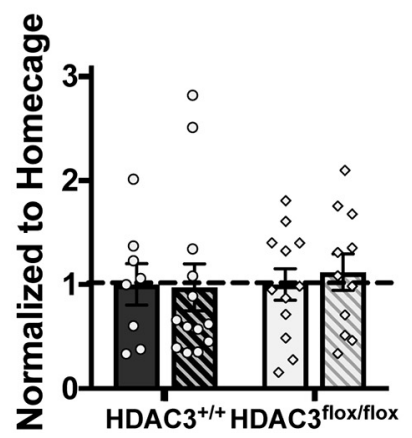

F Nr4a2 mRNA

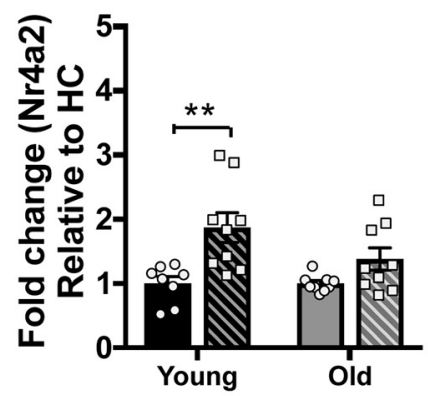

OLM

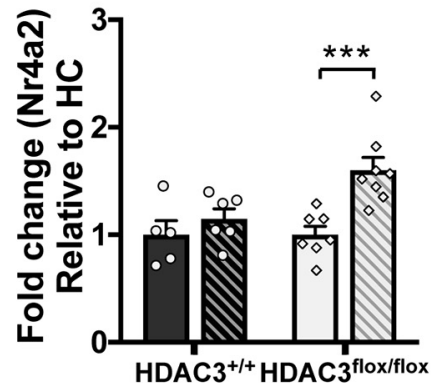

H H4K8Ac IP: Nr4a2 promoter

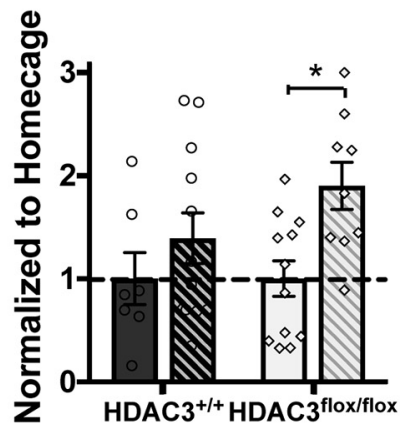

Figure 3. HDAC3 negatively regulates both $\mathrm{Nr} 4 \mathrm{a} 2 \mathrm{mRNA}$ and acetylation at the Nr4a2 promoter in the aged mouse hippocampus. (A) Experimental design for $\boldsymbol{B}-\boldsymbol{C}$. Young and old mice were sacrificed $\mathrm{th}$ after $0 \mathrm{LM}$ training or were sacrificed from HC. (B) Nr4a1 mRNA was significantly increased in the dorsal hippocampus of both young and old mice following OLM. (C) Nr4a2 mRNA was upregulated in the dorsal hippocampus of young mice after OLM but failed to increase in old mice. (D) Experimental design for E-H. 18-month-old HDAC3 ${ }^{+/+}$and HDAC3 $3^{\text {flox/flox }}$ mice injected with AAV-CaMKII-Cre into the dorsal hippocampus were sacrificed $1 \mathrm{~h}$ after $0 \mathrm{LM}$ training (OLM) or were sacrificed from HC. (E) Nr4a1 mRNA was significantly increased in the dorsal hippocampus of both aged HDAC3 ${ }^{+/+}$and aged HDAC ${ }^{\text {flox/flox }}$ mice following OLM. (F) Nr4a2 mRNA failed to increase after OLM in aged $\mathrm{HDAC}^{+/+}$mice, but is significantly upregulated by $0 \mathrm{LM}$ in the absence of HDAC3 in aged HDAC3 $3^{\text {flox/flox }}$ mice. (G) Occupancy of H4K8Ac at the Nr4a1 promoter was not affected by OLM training in either HDAC3 ${ }^{+/+}$or HDAC3 flox/flox mice. $(\boldsymbol{H})$ Occupancy of $\mathrm{H} 4 \mathrm{~K} 8 \mathrm{Ac}$ at the Nr4a2 promoter was not altered by learning in $\mathrm{HDAC}^{+/+}$mice, but $\mathrm{H} 4 \mathrm{~K} 8 \mathrm{Ac}$ was enriched at the Nr4a2 promoter

HDAC3 negatively regulates expression of $\mathrm{Nr} 4 \mathrm{a} 2$ and acetylation at the

Nr4a2 promoter

Next, we aimed to test whether the repressive histone deacetylase HDAC3 might contribute to this observed impairment in learning-induced $\mathrm{Nr} 4 \mathrm{a} 2$ in the old hippocampus. Numerous studies have suggested that the Nr4a1 and Nr4a2 are critical targets of HDAC3 during learning and previous work has demonstrated that deletion of HDAC3 restores learninginduced increases in $\mathrm{Nr} 4 \mathrm{a} 2$ in the dorsal hippocampus. Further, in a previous RNA sequencing experiment from our laboratory (Kwapis et al., 2018), Nr4al was one of only four genes identified as impaired with age, but restored by HDAC3 deletion. Finally, work has shown that knockdown of Nr4a2 prevents the memoryenhancing effects of HDAC3 deletion (McQuown et al., 2011). Together, these studies suggest that Nr4a1 and Nr4a2 are key targets of HDAC3; possibly serving as a mechanism through which HDAC3 negatively regulates long-term hippocampal memory.

To determine whether HDAC3mediated regulation of $\mathrm{Nr} 4 a 1$ and $\mathrm{Nr} 4 a 2$ contributes to age-related memory impairments, we used the HDAC3 $3^{\text {flox/flox }}$ mouse line, which allowed us to create focal genetic deletions of HDAC3 in the dorsal hippocampus of aged, 18-monthold mice by locally infusing AAVCaMKII-Cre. Previous work from our laboratory has demonstrated that 18month-old HDAC $3^{+/+}$mice show severe deficits in OLM following 10 min training and deleting HDAC3 in the dorsal hippocampus of HDAC $3^{\text {flox/flox }}$ littermates is sufficient to ameliorate age-related impairments in both long-term memory and synaptic plasticity (Kwapis et al., 2018). To determine whether HDAC3-mediated repression of Nr4a1 and Nr4a2 contributes to age-related impairments in OLM, we used RT-qPCR and ChIP-qPCR in 18month-old $\mathrm{HDAC}^{+/+}$and $\mathrm{HDAC}^{\text {flox/llox }}$ littermates (Fig. 3D).

We first examined expression of $\mathrm{Nr} 4 \mathrm{al}$ and Nr4a2 mRNA in the dorsal hippocampus. Nr4a1 mRNA was induced by learning in both $\mathrm{HDAC}^{+/+}$and $\mathrm{HDAC}^{\text {flox/flox }}$ mice [Fig. 3E; two-way ANOVA, significant main effect of training: $F_{(1,23)}=17.35, p=0.0004$; but no significant effect of genotype or signifi-

\footnotetext{
following OLM training in aged $\mathrm{HDAC} 3^{\text {floxflox }}$ mice. All data are
} shown as mean \pm SEM. ${ }^{*} p<0.05,{ }^{* *} p<0.01,{ }^{* * *} p<0.001$. 
cant interaction: $n=6(2 \mathrm{~F}), 7(3 \mathrm{~F}), 7(4 \mathrm{~F}), 7(5 \mathrm{~F})]$. Despite showing age-related impairments in long-term memory, these 18-monthold mice show successful learning-induced increases in Nr4al, even in the presence of HDAC3, consistent with the results of Figure 3B. Nr4a2 mRNA, on the other hand, failed to be induced by learning in aged $\mathrm{HDAC}^{+/+}$mice [Fig. $3 F$; two-way ANOVA, significant training by genotype interaction: $F_{(1,22)}=4.424, p=$ 0.047 ; Sidak's post hoc comparing HDAC $3^{+/+} \mathrm{HC}$ vs HDAC $3^{+/+}$ OLM: $p=0.606, n=5(1 \mathrm{~F}), 6(2 \mathrm{~F})]$, consistent with the agerelated impairment in $\mathrm{Nr} 4 \mathrm{a} 2$ observed in Figure 3C. For aged $\mathrm{HDAC}^{\text {flox/flox }}$ mice, however, learning-induced increases were restored [Sidak's post hoc comparing HDAC $3^{\text {flox/flox }} \mathrm{HC}$ vs HDAC3 ${ }^{\text {flox/flox }}$ OLM: $\left.p=0.0006, n=7(4 \mathrm{~F}), 8(5 \mathrm{~F})\right]$. This indicates that the failed induction of Nr4a2 in age-impaired mice is reversed by HDAC3 deletion, consistent with previous reports showing that Nr4a2 is an important target of HDAC3-mediated repression. Together, these results suggest that $\mathrm{Nr} 4 a 2$, but not $\mathrm{Nr} 4 a 1$ expression is repressed by HDAC 3 in the aged brain, possibly contributing to age-related memory impairments.

Next, to determine whether deletion of HDAC3 restores $\mathrm{Nr} 4 \mathrm{a} 2$ expression by enabling learning-induced histone acetylation, we used ChIP-qPCR to measure acetylation of histone 4 , lysine 8 (H4K8ac) at the $\mathrm{Nr} 4 a 1$ and $\mathrm{Nr} 4 \mathrm{a} 2$ promoters. $\mathrm{H} 4 \mathrm{~K} 8 \mathrm{Ac}$ is associated with active transcription (Kouzarides, 2007), is a target of HDAC3 (McQuown et al., 2011; Malvaez et al., 2013; Kwapis et al., 2017), and is enriched at multiple memory-relevant genes following a learning event, including Nr4a2 (Malvaez et al., 2013; Rogge et al., 2013; Kwapis et al., 2017). H4K8Ac levels at the Nr4a1 promoter were not altered by learning in either $\mathrm{HDAC}^{+/+}$or HDAC $3^{\text {flox/llox }}$ mice [Fig. $3 G$; two-way ANOVA, no significant main effects of genotype or training and no significant interaction: $n=8(4 \mathrm{~F}), 13(5 \mathrm{~F}), 12(5 \mathrm{~F}), 11(6 \mathrm{~F})]$. For Nr4a2, on the other hand, we observed no change in H4K8Ac occupancy in response to learning in $\mathrm{HDAC}^{+/+}$mice, but in $\mathrm{HDAC} 3^{\text {flox/flox }}$ mice, learning enriched $\mathrm{H} 4 \mathrm{~K} 8 \mathrm{Ac}$ at the $\mathrm{Nr} 4 \mathrm{a} 2$ promoter [Fig. $3 \mathrm{H}$; two-way ANOVA, significant main effect of training: $F_{(1,36)}=7.83, p=0.0082$, Sidak's post hoc tests: $\mathrm{HDAC}^{+/+} \mathrm{HC}$ vs HDAC3 ${ }^{+/+}$OLM, $p=0.444$; $\mathrm{HDAC}^{\text {flox/flox }} \mathrm{HC}$ vs HDAC3 $3^{\text {flox/flox }} \mathrm{OLM}, p=0.014, n=$ $7(5 \mathrm{~F}), 12(4 \mathrm{~F}), 12(5 \mathrm{~F}), 9(6 \mathrm{~F})]$. These results suggest that HDAC3 limits expression of Nr4a2, but not Nr4a1, in the aged hippocampus by restricting learning-induced histone acetylation at its promoter.

\section{Overexpression of either Nr4a1 or $\mathrm{Nr} 4 \mathrm{a} 2$ in the aged hippocampus improves memory for OLM.}

Our results demonstrate that impairments in $\mathrm{Nr} 4 \mathrm{a} 2$ expression accompany cognitive deficits in aged mice and rats. Further, HDAC 3 contributes to this age-related repression of Nr4a2, as focal deletion of HDAC3 restores both Nr4a2 expression (Fig. $3 F$ ) and long-term memory formation (Kwapis et al., 2018) in aged mice. This suggests that Nr4a2 may be a key mechanism through which HDAC3-mediated regulation represses memory formation in the aged brain. To determine whether overexpression of Nr4a2 is sufficient to ameliorate age-related impairments in hippocampal memory formation, we locally injected a virus expressing the full-length $\mathrm{Nr} 4 \mathrm{a} 2$ construct with a V5 epitope tag (AAV-v5-Nr4a2). Additionally, as Nr4a2 heterodimerizes with Nr4a1 to synergistically promote transcription at NurRE (Nur response element) sequences, we also locally injected a virus expressing full-length Nr4al tagged with HA (AAV-HA-Nr4al) either alone or in combination with AAV-v5-Nr4a2. Two weeks after injection, when viruses are maximally expressed (Mc-
Quown et al., 2011; Kwapis et al., 2018), we trained animals in the hippocampus-dependent OLM task followed by the ORM task, which does not require the dorsal hippocampus for retrieval (Vogel-Ciernia et al., 2013; Fig. 4A).

To confirm that the AAVs expressed appropriately following injection in the dorsal hippocampus, we measured immunoreactivity to the V5 and HA epitope tags for each group. Although visualization of the HA epitope tag was unsuccessful, we observed robust expression of V5 in mice injected with either AAV-V5$\mathrm{Nr} 4 \mathrm{a} 2$ alone or both AAV-V5-Nr4a2 and AAV-HA-Nr4a1 (Fig. $4 B$ ). V5 labeling was observed throughout areas CA1 and CA3 of the dorsal hippocampus, similar to the spread previously observed in our laboratory following intrahippocampal AAV2.1 (Barrett et al., 2011; McQuown et al., 2011; Vogel-Ciernia et al., 2013; Kwapis et al., 2017, 2018). Because we were unable to verify the expression of AAV-HA-Nr4al with immunofluorescence (the HA epitope may be obscured), we used RT-qPCR to confirm the presence of each virus in hippocampal tissue from each animal using primers against both the endogenous and exogenous transcripts. First, primers against the HA region of the HA-Nr4al transcript confirmed significantly higher expression of $H A$ Nr4al in the group infused with AAV-HA-Nr4al (one-way ANOVA: $F_{(3,32)}=15.15, p<0.0001$; Sidak's post hoc comparing AAV-EV to AAV-HA-Nr4a1: $p<0.0001$ ) or both viruses (Sidak's post hoc comparing AAV-EV to both, $p=0.0002$ ) compared with EV controls (Fig. $4 C ; n=10,9,9,8$, all males). Similarly, primers against the v5 region of $v 5-\mathrm{Nr} 4 a 2$ showed significantly higher expression in the groups infused with either AAV-v5-Nr4a2 (one-way ANOVA: $F_{(3,32)}=237.2, p<0.0001$; Sidak's post hoc comparing AAV-EV to AAV-V5-Nr4a2: $p<$ 0.0001) or both viruses (Sidak's post hoc comparing AAV-EV to both, $p<0.0001$ ) compared with EV controls (Fig. $4 D ; n=$ $10,9,9,8$, all males). Next, we designed primers targeting the endogenous $\mathrm{Nr} 4 a 1$ and Nr4a2 transcripts to determine whether these viruses produce overexpression of each target. Indeed, we observed significantly higher expression of Nr4al in the group infused with AAV-HA-Nr4al (one-way ANOVA: $F_{(3,32)}=6.936$, $p=0.001$; Sidak's post hoc comparing AAV-EV to AAV-V5$\mathrm{Nr} 4 \mathrm{al}, p=0.0012$ ) or both viruses (Sidak's post hoc comparing AAV-EV to both, $p=0.0175$ ) compared with EV controls (Fig. $4 E ; n=10,9,9,8$, all males). Similarly, for Nr4a2, we found significantly higher expression in both groups infused with AAVv5-Nr4a2 compared with EV controls (Fig. 4F; one-way ANOVA, $F_{(3,32)}=237.2, p<0.0001$; Sidak's post hoc comparing AAV-EV to AAV-Nr4a2, $p<0.0001$; Sidak's post hoc comparing AAV-EV to both, $p<0.0001 n=10,9,9,8$, all males). The viruses therefore appropriately overexpressed $\mathrm{Nr} 4 a 1, \mathrm{Nr} 4 a 2$, or both transcripts in the dorsal hippocampus.

To determine whether overexpression of Nr4a1, Nr4a2, or both transcripts in the dorsal hippocampus improves hippocampus-dependent memory, we injected the viruses locally into the dorsal hippocampus 2 weeks before behavior (Fig. 4A). Mice infused with AAV-HA-Nr4a1, AAV-v5-Nr4a2, or both viruses showed significantly improved memory for OLM at test relative to $\mathrm{EV}$ controls (Fig. 4G; one-way ANOVA: $F_{(3,28)}=$ $6.826, p=0.0014$; Sidak's post hoc comparing AAV-EV to AAVHA-Nr4a1: $p=0.003$; AAV-EV to AAV-V5-Nr4a2: $p=0.011$; AAV-EV to both: $p=0.003, n=10,5,11,6$, all males) with no group differences observed in total exploration time (one-way ANOVA: $F_{(3,28)}=0.689, p=0.566$; Fig. $\left.4 H\right)$. Overexpression of $\mathrm{Nr} 4 a 1, \mathrm{Nr} 4 a 2$, or both transcripts in the dorsal hippocampus was therefore sufficient to ameliorate age-related impairments in long-term hippocampus-dependent memory. 
A

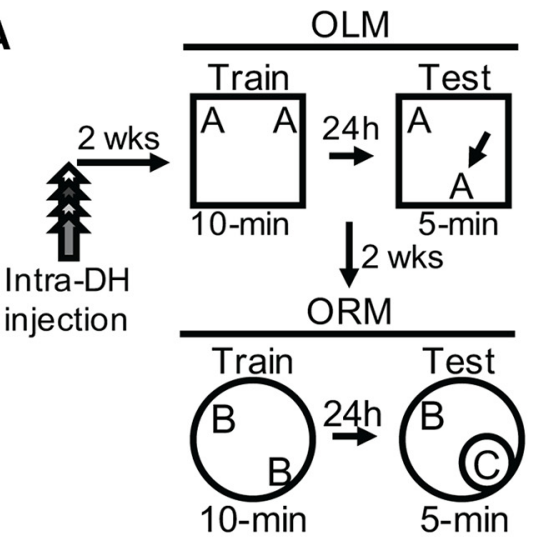

B

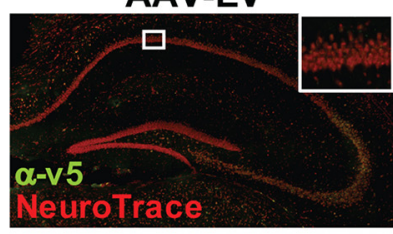

AAV-V5-Nr4a2

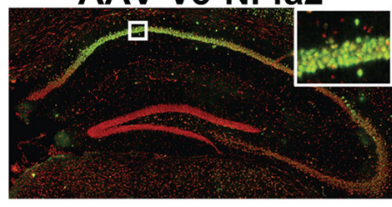

AAV-HA-Nr4a1

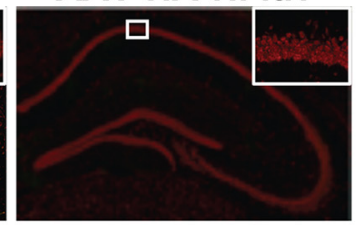

Both

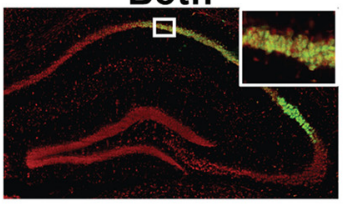

C HA-Nr4a1 mRNA
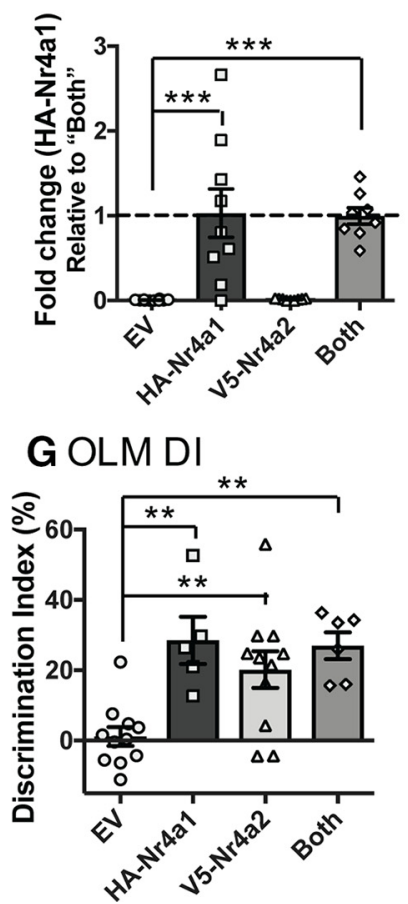

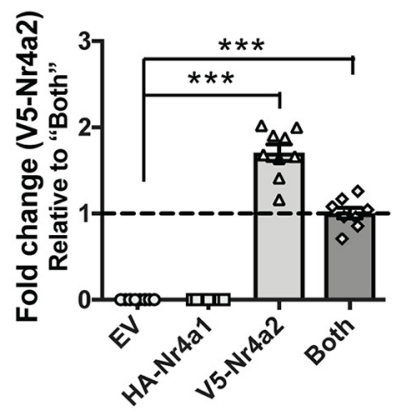

H OLM Exploration

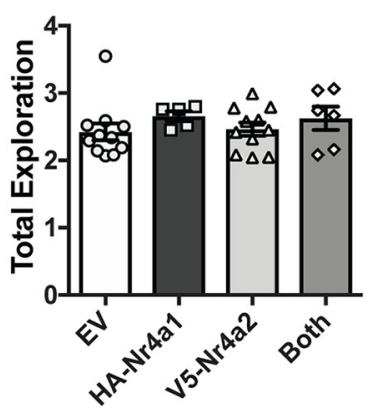

E Nr4a1mRNA
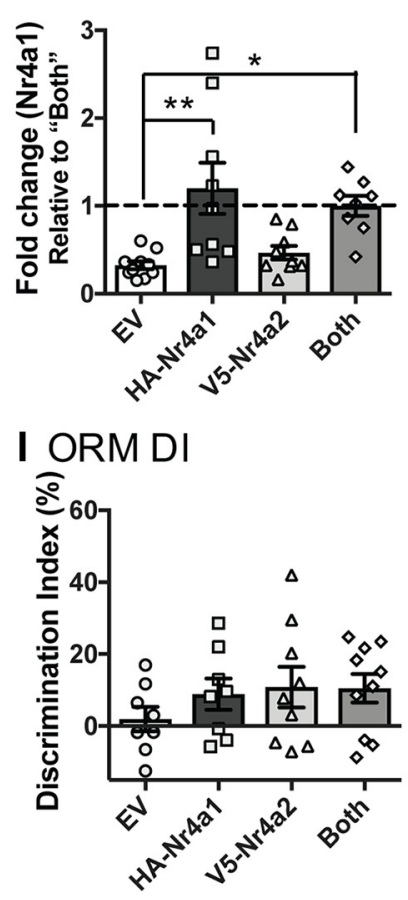

F Nr4a2mRNA

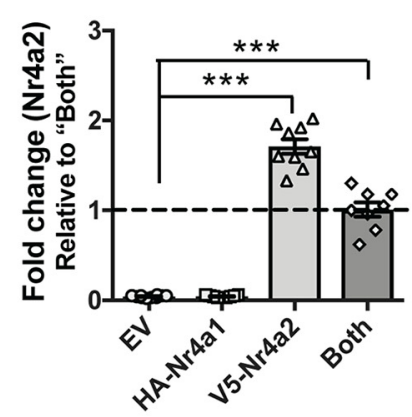

J ORM Exploration

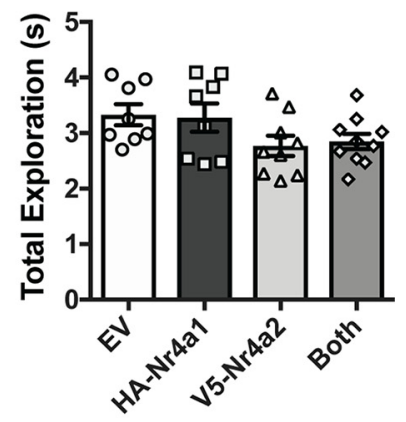

Figure 4. Hippocampal overexpression of Nr4a1, Nr4a2, or both transcripts enhances OLM. A, Experimental design. Following injection of AAV-EV, AAV-HA-Nr4a1, AAV-V5-Nr4a2, or both AAV-HA-Nr4a1 and AAV-V5-Nr4a2, mice were trained and tested in 0LM. A subset of these animals was then trained in ORM. $\boldsymbol{B}$, Representative immunofluorescence images showing expression of the V5 epitope tag (green) in the dorsal hippocampus following injection of EV, AAV-HA-Nr4a1, AAV-V5-Nr4a2, or both viruses. V5 labeling was observed in animals injected with AAV-V5-Nr4a2 or both viruses. Neurons are counterstained with NeuroTrace (red), a fluorescent Nissl stain. Immunolabeling of the HA epitope tag on AAV-HA-Nr4a1 was unsuccessful and is not shown. $\boldsymbol{C}-\boldsymbol{F}$, RT-qPCR verification of viral expression. $\boldsymbol{C}$, The HA-Nr4a1 transcript was expressed in the groups infused with AAV-HA-Nr4a1 and both viruses. D, V5-Nr4a2 was expressed in groups infused with AAV-v5$\mathrm{Nr} 4 \mathrm{a} 2$ or both viruses. $\boldsymbol{E}$, Endogenous Nr4a1 mRNA was expressed at significantly higher levels in groups injected with either AAV-HA-Nr4a1 or both viruses. $\boldsymbol{F}$, Endogenous Nr4a2 mRNA was expressed at significantly higher levels in group injected with either AAV-v5-Nr4a2 or both viruses. G, Mice injected with either AAV-HA-Nr4a1, AAV-v5-Nr4a2, or both viruses showed significantly better memory for OLM than AAV-EV controls. $\boldsymbol{H}$, All groups showed similar levels of total object exploration during the OLM test. $\boldsymbol{I}$, Hippocampal infusion of the viruses did not improve memory for ORM. J, All groups showed similar levels of total object exploration during the ORM test. All data are shown as mean \pm SEM. ${ }^{*} p<0.05,{ }^{* *} p<0.01,{ }^{* * *} p<0.001$.

Finally, we tested whether local overexpression of Nr4al or Nr4a2 affects ORM, in which one of the trained objects is replaced with a novel object at test (Fig. 4A). All four groups of aged mice showed poor memory for ORM, with no improvement observed following overexpression of $\mathrm{Nr} 4 a 1, \mathrm{Nr} 4 a 2$, or both transcripts (Fig. 4I; one-way ANOVA: $F_{(3,31)}=0.8231, p=0.4911$, $n=8,8,9,10$, all male). We also saw no significant difference between groups in total exploration time (one-way ANOVA: $F_{(3,31)}=2.284, p=0.0984$; Fig. $\left.4 J\right)$. Local overexpression of either Nr4a1 or Nr4a2 therefore improved memory for a hippocampus-dependent task (OLM) in aged mice without ameliorating memory impairments in a hippocampus-independent task (ORM).

\section{Discussion}

This study demonstrates that repression of Nr4a2 contributes to age-related impairments in memory, in addition to its known role in supporting memory formation in the young brain. Using a cross-species approach, we found that learning-induced increases in hippocampal $\mathrm{Nr} 4 \mathrm{a} 2$ expression are impaired in aged mice and rats with cognitive deficits. In comparison, $\mathrm{Nr} 4 a 1$ expression was not consistently affected by age across species, nor indicative of performance related to memory. Age-related impairments in $\mathrm{Nr} 4 \mathrm{a} 2$ expression and $\mathrm{H} 4 \mathrm{~K} 8$ acetylation at the $\mathrm{Nr} 4 \mathrm{a} 2$ promoter were reversed with local HDAC3 deletion, suggesting that HDAC3 typically restricts $\mathrm{Nr} 4 a 2$ expression in the 
aged brain by promoting a closed chromatin state at the $\mathrm{Nr} 4 \mathrm{a} 2$ promoter. Finally, we found that local overexpression of either Nr4a1, Nr4a2, or both transcripts within the dorsal hippocampus ameliorates memory impairments in aged mice. Together, these results indicate that epigenetic repression of $\mathrm{Nr} 4 a 2$ contributes to age-related memory impairments, but overexpression of either $\mathrm{Nr} 4 a 2$ or its binding partner Nr4a1 can ameliorate these memory deficits.

Here, we chose to use a cross-species approach to exploit the different strengths of two different rodent models of age-related memory decline. First, we used a well characterized rat model that is sensitive to individual differences in age-related memory performance (Gallagher et al., 2006). Using an outbred population of Long-Evans rats, this model detects a range of cognitive abilities in the aged rat that allows AU rats to be assessed separately from AI rats in the same population. Using this approach, we identified $\mathrm{Nr} 4 \mathrm{a} 2$ as a potential mechanism that supports preserved cognitive function; impairments in $\mathrm{Nr} 4 \mathrm{a} 2$ expression were only observed in the cognitively impaired aged group of rats. To complement this approach, we next used a mouse model to enable genetic manipulations of both HDAC3 (a known regulatory mechanism of Nr4a2; McQuown et al., 2011; Malvaez et al., 2013; Rogge et al., 2013; Kwapis et al., 2017) and Nr4a2 itself in the aging brain. This confirmed that HDAC 3 represses Nr4a2 expression in the aged hippocampus and demonstrated that overexpression of the Nr4a gene family can improve memory in the aged mouse. Thus, Nr4a2 is a prime candidate gene that may support preserved cognitive function in aging, with HDAC3-mediated deficits in Nr4a2 likely contributing to the impaired memory observed in AI rats and aged mice.

Although the cross-species approach provided converging lines of evidence that Nr4a2 plays a critical role in age-related memory decline, our findings for Nr4al across species were less consistent. In aged rats, Nr4al was not significantly increased by OLM training, even in AU rats, although there was a trend toward a learning-induced increase in this group. For aged mice, on the other hand, OLM training triggered a significant increase in $\mathrm{Nr} 4 a 1$ in two separate groups of old wild-type mice (Fig. $3 B, E$ ), even though aged mice show impaired long-term memory using this same task under identical training and testing procedures (Kwapis et al., 2018). There are a number of possible reasons for these disparate results across species, including genetic variability (the rats are outbred whereas the mice are inbred), differences in the severity of age-related memory impairments (aged rats show more variability in memory performance than aged mice), and slight procedural differences in the task across species. In any case, the consistent repression of $\mathrm{Nr} 4 a 2$ in age-impaired animals across this cross-species variability strengthens the likelihood that $\mathrm{Nr} 4 \mathrm{a} 2$ is a key mechanism contributing to cognitive performance in old age.

Additionally, our finding that aged mice show normal learning-induced increases in Nr4al (Fig. 3B,E) was somewhat surprising, as a previous study from our laboratory had identified $\mathrm{Nr} 4 a 1$ as one of a small subset of genes that fit the criteria of being induced by learning in the young mouse hippocampus, impaired in the old mouse hippocampus, but induced by learning in the old hippocampus in mice with a conditional deletion of HDAC3 (Kwapis et al., 2018). Although unexpected, there are a few possible reasons for the discrepancy between this study and our previous report. First, it is possible that Nr4a1 expression was falsely identified in our previous RNA sequencing study as being impaired with age but restored following HDAC3 deletion. As Pe- riod1 (Per1) was the focus of our previous paper, we did not use RT-qPCR to confirm the pattern of Nr4a1 expression observed in our sequencing data. Further, in our RNA-seq study, Nr4a2 expression appeared to follow the same general pattern as Nr4al (Fig. 4C; Kwapis et al., 2018), but the fold-change was not sufficiently robust to be identified in our unbiased sequencing analysis. Second, it seems that the variability in Nr4a1 expression may have contributed to the inconsistent results across experiments, possibly because $\mathrm{Nr} 4 \mathrm{a} 1$ expression levels may reflect the severity of cognitive impairment in mice. We observed high variability in Nr4a1 expression in both wild-type and HDAC $3^{\text {flox/flox }}$ mice, consistent with the relatively high variability observed in our AU rat group (Fig. 2B). Here, we show in two independent groups of wild-type mice (Fig. $3 B, E$ ) that Nr4a1 expression is readily induced by OLM training in the old hippocampus. Thus, although the results of our previous RNA sequencing study suggested that aging impairs learning-induced $\mathrm{Nr} 4 a \mathrm{l}$ expression, the current study demonstrates that learning-induced $\mathrm{Nr} 4 a 1$ expression is intact in aged mice.

Although we initially hypothesized that HDAC3-mediated repression of both Nr4a1 and Nr4a2 would contribute to agerelated memory impairments, we only observed consistent repression of Nr4a2 in the aged hippocampus following OLM. Further, HDAC3 deletion failed to restore expression of $\mathrm{Nr} 4 \mathrm{a} 1$ or acetylation at the Nr4al promoter in the dorsal hippocampus of aged mice. Nonetheless, we found that overexpression of $\mathrm{Nr} 4 a \mathrm{l}$, like overexpression of $\mathrm{Nr} 4 \mathrm{a} 2$, was able to ameliorate age-related impairments in memory performance for OLM. There are a few potential reasons for our observed memory rescue following overexpression of either Nr4a1 or Nr4a2. As we observed no synergistic effects in response to overexpression of both transcripts, it is possible that at sufficient levels, $\mathrm{Nr} 4 a 1$ is capable of driving transcription of key Nr4a2 target genes to restore memory function. Indeed, NR4A1 and NR4A2 bind identical sequences in DNA to activate transcription of target genes (Wilson et al., 1991; Paulsen et al., 1995; Zetterström et al., 1996; Cheng et al., 1997; Maira et al., 1999) with their unique functions coming from differential activation through post-translational modifications and heterogeneous binding partners (Hawk et al., 2012). Similarly, it is possible that overexpression of NR4A1 improves memory in the aged brain by improving NR4A2's stability or targeting, as these molecules are known to heterodimerize to synergistically drive transcription (Maira et al., 1999; Hawk and Abel, 2011). Future work will be necessary to determine the mechanism through which $\mathrm{Nr} 4 a 1$ and $\mathrm{Nr} 4 a 2$ overexpression can improve memory formation in aged mice, including investigation of putative Nr4a family gene targets such as Bdnf, Fosl2, and Pak6 (Hawk et al., 2012).

The previous RNA sequencing study from our laboratory also identified the circadian gene Perl as a downstream target of HDAC3 that, like Nr4a2, is repressed in the aging brain (Kwapis et al., 2018). Although it is unclear how (or whether) these agerelated changes in Perl and the Nr4a gene family are related, overexpressing either Per1, Nr4a1, or Nr4a2 in the dorsal hippocampus is sufficient to ameliorate age-related impairments in long-term memory formation. In addition to being negatively regulated by HDAC3, each of these genes can be directly regulated by CREB, a transcription factor that is critical for long-term memory formation. It is possible that CREB promotes the expression of both Per1 and the Nr4a genes following HDAC3 removal after a learning event. Another possibility is that Perl may gate the likelihood of learning-induced $\mathrm{Nr} 4 a$ gene expression over the $24 \mathrm{~h}$ day, possibly by restricting or enabling CREB phos- 
phorylation at different times of day (Rawashdeh et al., 2014, 2016) to affect Nr4a gene expression. Finally, it is possible that Nr4a1 and Nr4a2, which are transcription factors themselves, may be upstream of Per1, although this was not identified as a putative Nr4a target gene in previous work (Hawk et al., 2012). Understanding how age-related changes in Nr4a1 and $\mathrm{Nr} 4 a 2$ interact with changes in Per1 and other genes implicated in agerelated memory decline will be a major goal for future research.

In conclusion, we found that learning-induced $\mathrm{Nr} 4 \mathrm{a} 2$, but not $N r 4 a 1$, is impaired in the dorsal hippocampus of rats and mice with age-related memory deficits. Further, Nr4a2 expression is limited in the aged brain through the repressive histone deacetylase HDAC3. Finally, overexpression of either Nr4a1, Nr4a2, or both transcripts in the dorsal hippocampus is sufficient to ameliorate age-related impairments in hippocampal memory formation. Together, these results show that HDAC3-mediated repression of Nr4a2 may contribute to age-related cognitive impairments. Treatments that enhance expression or activity of the Nr4a gene family may therefore be an effective strategy to improve memory in old age.

\section{References}

Alaghband Y, Kwapis JL, López AJ, White AO, Aimiuwu OV, Al-Kachak A, Bodinayake KK, Oparaugo NC, Dang R, Astarabadi M, Matheos DP, Wood MA (2017) Distinct roles for the deacetylase domain of HDAC3 in the hippocampus and medial prefrontal cortex in the formation and extinction of memory. Neurobiol Learn Mem 145:94-104.

Alaghband Y, Kramár E, Kwapis JL, Kim ES, Hemstedt TJ, López AJ, White AO, Al-Kachak A, Aimiuwu OV, Bodinayake KK, Oparaugo NC, Han J, Lattal KM, Wood MA (2018) CREST in the nucleus accumbens core regulates cocaine conditioned place preference, cocaine-seeking behavior, and synaptic plasticity. J Neurosci 38:9514-9526.

Alberini CM (2009) Transcription factors in long-term memory and synaptic plasticity. Physiol Rev 89:121-145.

Ash JA, Lu H, Taxier LR, Long JM, Yang Y, Stein EA, Rapp PR (2016) Functional connectivity with the retrosplenial cortex predicts cognitive aging in rats. Proc Natl Acad Sci U S A 113:12286-12291.

Barrett RM, Malvaez M, Kramar E, Matheos DP, Arrizon A, Cabrera SM, Lynch G, Greene RW, Wood MA (2011) Hippocampal focal knock-out of CBP affects specific histone modifications, long-term potentiation, and long-term memory. Neuropsychopharmacology 36:1545-1556.

Benito E, Urbanke H, Ramachandran B, Barth J, Halder R, Awasthi A, Jain G, Capece V, Burkhardt S, Navarro-Sala M, Nagarajan S, Schütz AL, Johnsen SA, Bonn S, Lührmann R, Dean C, Fischer A (2015) HDAC inhibitordependent transcriptome and memory reinstatement in cognitive decline models. J Clin Invest 125:3572-3584.

Bieszczad KM, Bechay K, Rusche JR, Jacques V, Kudugunti S, Miao W, Weinberger NM, McGaugh JL, Wood MA (2015) Histone deacetylase inhibition via RGFP966 releases the brakes on sensory cortical plasticity and the specificity of memory formation. J Neurosci 35:13124-13132.

Bridi MS, Hawk JD, Chatterjee S, Safe S, Abel T (2017) Pharmacological activators of the NR4A nuclear receptors enhance LTP in a CREB/CBPdependent manner. Neuropsychopharmacology 42:1243-1253.

Castellano JF, Fletcher BR, Kelley-Bell B, Kim DH, Gallagher M, Rapp PR (2012) Age-related memory impairment is associated with disrupted multivariate epigenetic coordination in the hippocampus. PloS one 7:e33249.

Cheng LE, Chan FK, Cado D, Winoto A (1997) Functional redundancy of the Nur77 and nor-1 orphan steroid receptors in T-cell apoptosis. EMBO J 16:1865-1875.

Gallagher M, Burwell R, Burchinal M (1993) Severity of spatial learning impairment in aging: development of a learning index for performance in the Morris water maze. Behav Neurosci 107:618-626.

Gallagher M, Colantuoni C, Eichenbaum H, Haberman RP, Rapp PR, Tanila $\mathrm{H}$, Wilson IA (2006) Individual differences in neurocognitive aging of the medial temporal lobe. Age 28:221-233.

Hawk JD, Abel T (2011) The role of NR4A transcription factors in memory formation. Brain Res Bull 85:21-29.

Hawk JD, Bookout AL, Poplawski SG, Bridi M, Rao AJ, Sulewski ME, Kroener
BT, Manglesdorf DJ, Abel T (2012) NR4A nuclear receptors support memory enhancement by histone deacetylase inhibitors. J Clin Invest 122:3593-3602.

Jarome TJ, Thomas JS, Lubin FD (2014) The epigenetic basis of memory formation and storage. Prog Mol Biol Transl Sci 128:1-27.

Koh MT, Haberman RP, Foti S, McCown TJ, Gallagher M (2010) Treatment strategies targeting excess hippocampal activity benefit aged rats with cognitive impairment. Neuropsychopharmacology 35:1016-1025.

Kouzarides T (2007) Chromatin modifications and their function. Cell 128:693-705.

Kwapis JL, Wood MA (2014) Epigenetic mechanisms in fear conditioning: implications for treating post-traumatic stress disorder. Trends Neurosci 37:706-720.

Kwapis JL, Alaghband Y, López AJ, White AO, Campbell RR, Dang RT, Rhee D, Tran AV, Carl AE, Matheos DP, Wood MA (2017) Context and auditory fear are differentially regulated by HDAC 3 activity in the lateral and basal subnuclei of the amygdala. Neuropsychopharmacology 42:12841294.

Kwapis JL, Alaghband Y, Kramár EA, López AJ, Vogel Ciernia A, White AO, Shu G, Rhee D, Michael CM, Montellier E, Liu Y, Magnan CN, Chen S, Sassone-Corsi P, Baldi P, Matheos DP, Wood MA (2018) Epigenetic regulation of the circadian gene Perl contributes to age-related changes in hippocampal memory. Nat Commun 9:3323.

Leighton LJ, Zhao Q, Li X, Dai C, Marshall PR, Liu S, Wang Y, Zajaczkowski EL, Khandelwal N, Kumar A, Bredy TW, Wei W (2018) A functional role for the epigenetic regulator ING1 in activity-induced gene expression in primary cortical neurons. Neuroscience 369:248-260.

Levenson JM, O'Riordan KJ, Brown KD, Trinh MA, Molfese DL, Sweatt JD (2004) Regulation of histone acetylation during memory formation in the hippocampus. J Biol Chem 279:40545-40559.

Lopez AJ, Jia Y, White AO, Kwapis JL, Espinoza M, Hwang P, Campbell R, Alaghband Y, Chitnis O, Matheos DP, Lynch G, Wood MA (2018) Medial habenula cholinergic signaling regulates cocaine-associated relapse-like behavior. Addict Biol 24:403-413.

Maira M, Martens C, Philips A, Drouin J (1999) Heterodimerization between members of the nur subfamily of orphan nuclear receptors as a novel mechanism for gene activation. Mol Cell Biol 19:7549-7557.

Malvaez M, McQuown SC, Rogge GA, Astarabadi M, Jacques V, Carreiro S, Rusche JR, Wood MA (2013) HDAC3-selective inhibitor enhances extinction of cocaine-seeking behavior in a persistent manner. Proc Natl Acad Sci U S A 110:2647-2652.

McNulty SE, Barrett RM, Vogel-Ciernia A, Malvaez M, Hernandez N, Davatolhagh MF, Matheos DP, Schiffman A, Wood MA (2012) Differential roles for $\mathrm{Nr} 4 \mathrm{a} 1$ and $\mathrm{Nr} 4 \mathrm{a} 2$ in object location vs. object recognition longterm memory. Learn Mem 19:588-592.

McQuown SC, Barrett RM, Matheos DP, Post RJ, Rogge GA, Alenghat T, Mullican SE, Jones S, Rusche JR, Lazar MA, Wood MA (2011) HDAC3 is a critical negative regulator of long-term memory formation. J Neurosci 31:764-774.

Paulsen RF, Granas K, Johnsen H, Rolseth V, Sterri S (1995) Three related brain nuclear receptors, NGFI-B, Nurr1, and NOR-1, as transcriptional activators. J Mol Neurosci 6:249-255.

Peleg S, Sananbenesi F, Zovoilis A, Burkhardt S, Bahari-Javan S, Agis-Balboa RC, Cota P, Wittnam JL, Gogol-Doering A, Opitz L, Salinas-Riester G, Dettenhofer M, Kang H, Farinelli L, Chen W, Fischer A (2010) Altered histone acetylation is associated with age-dependent memory impairment in mice. Science 328:753-756.

Peña de Ortiz S, Maldonado-Vlaar CS, Carrasquillo Y (2000) Hippocampal expression of the orphan nuclear receptor gene hzf-3/nurr 1 during spatial discrimination learning. Neurobiol Learn Mem 74:161-178.

Penner MR, Roth TL, Barnes CA, Sweatt JD (2010) An epigenetic hypothesis of aging-related cognitive dysfunction. Front Aging Neurosci 2:9.

Pfaffl MW (2001) A new mathematical model for relative quantification in real-time RT-PCR. Nucleic Acids Res 29:e45.

Pfaffl MW, Horgan GW, Dempfle L (2002) Relative expression software tool (REST) for group-wise comparison and statistical analysis of relative expression results in real-time PCR. Nucleic Acids Res 30:e36.

Rapp PR, Gallagher M (1996) Preserved neuron number in the hippocampus of aged rats with spatial learning deficits. Proc Natl Acad Sci U S A 93:9926-9930.

Rawashdeh O, Jilg A, Jedlicka P, Slawska J, Thomas L, Saade A, Schwarzacher 
SW, Stehle JH (2014) PERIOD1 coordinates hippocampal rhythms and memory processing with daytime. Hippocampus 24:712-723.

Rawashdeh O, Jilg A, Maronde E, Fahrenkrug J, Stehle JH (2016) Period1 gates the circadian modulation of memory-relevant signaling in mouse hippocampus by regulating the nuclear shuttling of the CREB kinase pP90RSK. J Neurochem 138:731-745.

Reolon GK, Maurmann N, Werenicz A, Garcia VA, Schröder N, Wood MA, Roesler R (2011) Posttraining systemic administration of the histone deacetylase inhibitor sodium butyrate ameliorates aging-related memory decline in rats. Behav Brain Res 221:329-332.

Rogge GA, Singh H, Dang R, Wood MA (2013) HDAC3 is a negative regulator of cocaine-context-associated memory formation. J Neurosci 33:6623-6632.

Roozendaal B, Hernandez A, Cabrera SM, Hagewoud R, Malvaez M, Stefanko DP, Haettig J, Wood MA (2010) Membrane-associated glucocorticoid activity is necessary for modulation of long-term memory via chromatin modification. J Neurosci 30:5037-5046.

Sharma M, Shetty MS, Arumugam TV, Sajikumar S (2015) Histone deacetylase 3 inhibition re-establishes synaptic tagging and capture in aging through the activation of nuclear factor kappa B. Sci Rep 5:16616.

Spiegel AM, Koh MT, Vogt NM, Rapp PR, Gallagher M (2013) Hilar interneuron vulnerability distinguishes aged rats with memory impairment. J Comp Neurol 521:3508-3523.

Spiegel AM, Sewal AS, Rapp PR (2014) Epigenetic contributions to cognitive aging: disentangling mindspan and lifespan. Learn Mem 21:569-574.

Stefanko DP, Barrett RM, Ly AR, Reolon GK, Wood MA (2009) Modulation of long-term memory for object recognition via HDAC inhibition. Proc Natl Acad Sci U S A 106:9447-9452.

Tomás Pereira I, Coletta CE, Perez EV, Kim DH, Gallagher M, Goldberg IG,
Rapp PR (2013) CREB-binding protein levels in the rat hippocampus fail to predict chronological or cognitive aging. Neurobiol Aging 34:832-844.

Vecsey CG, Hawk JD, Lattal KM, Stein JM, Fabian SA, Attner MA, Cabrera SM, McDonough CB, Brindle PK, Abel T, Wood MA (2007) Histone deacetylase inhibitors enhance memory and synaptic plasticity via CREB: CBP-dependent transcriptional activation. J Neurosci 27:6128-6140.

Vogel-Ciernia A, Matheos DP, Barrett RM, Kramár EA, Azzawi S, Chen Y, Magnan CN, Zeller M, Sylvain A, Haettig J, Jia Y, Tran A, Dang R, Post RJ, Chabrier M, Babayan AH, Wu JI, Crabtree GR, Baldi P, Baram TZ, et al. (2013) The neuron-specific chromatin regulatory subunit BAF53b is necessary for synaptic plasticity and memory. Nat Neurosci 16:552-561.

Vogel-Ciernia A, Wood MA (2014) Examining object location and object recognition memory in mice. Curr Protoc Neurosci 69:8.31.1-17.

von Hertzen LS, Giese KP (2005) Memory reconsolidation engages only a subset of immediate-early genes induced during consolidation. J Neurosci 25:1935-1942.

White AO, Kramár EA, López AJ, Kwapis JL, Doan J, Saldana D, Davatolhagh MF, Alaghband Y, Blurton-Jones M, Matheos DP, Wood MA (2016) BDNF rescues BAF53b-dependent synaptic plasticity and cocaineassociated memory in the nucleus accumbens. Nat Commun 7:11725.

Wilson TE, Fahrner TJ, Johnston M, Milbrandt J (1991) Identification of the DNA binding site for NGFI-B by genetic selection in yeast. Science 252:1296-1300

Zetterström RH, Solomin L, Mitsiadis T, Olson L, Perlmann T (1996) Retinoid $\mathrm{X}$ receptor heterodimerization and developmental expression distinguish the orphan nuclear receptors NGFI-B, Nurr1, and Nor1. Mol Endocrinol 10:1656-1666. 